\title{
Transcranial magnetic stimulation for treating depression in elderly patients
}

\author{
This article was published in the following Dove Press journal: \\ Neuropsychiatric Disease and Treatment \\ 12 April 2013 \\ Number of times this article has been viewed
}

\section{Gokben Hizli Sayar \\ Eylem Ozten \\ Oguz Tan \\ Nevzat Tarhan}

Uskudar University, Neuropsychiatry Istanbul Hospital, Department of Psychiatry, Istanbul, Turkey
Correspondence: Gokben Hizli Sayar Department of Psychiatry, Uskudar University, Neuropsychiatry Istanbul Hospital,Alemdag Caddesi Site Yolu No 29 Umraniye, Istanbul, Turkey

Tel +902166330649

Fax +90216634 I250

Email gokben.hizlisayar@uskudar.edu.tr
Purpose: The aim of the study reported here was to examine the safety and effectiveness of high-frequency repetitive transcranial magnetic stimulation (rTMS) in elderly patients with depression.

Patients and methods: Sixty-five depressed elderly patients received rTMS over their left prefrontal cortex for 6 days per week, from Monday to Saturday, for 3 weeks. The rTMS intensity was set at $100 \%$ of the motor threshold and $25 \mathrm{~Hz}$ stimulation with a duration of 2 seconds and was delivered 20 times at 30 -second intervals. A full course comprised an average of 1000 magnetic pulses. Depression was rated using the Hamilton Depression Rating Scale (HAMD) before and after treatment. Response was defined as a 50\% reduction in HAMD score. Patients with HAMD scores $<8$ were considered to be in remission.

Results: The mean HAMD score for the study group decreased from $21.94 \pm 5.12$ before treatment to $11.28 \pm 4.56$ after rTMS $(P<0.001)$. Following the treatment period, $58.46 \%$ of the study group demonstrated significant mood improvement, as indexed by a reduction of more than $50 \%$ on the HAMD score. Nineteen of these 38 patients attained remission (HAMD score $<8$ ), while $41.54 \%$ of all study patients achieved a partial response. None of the patients had a worsened HAMD score at the end of the treatment. Treatment was generally well tolerated and no serious adverse effects were reported.

Conclusion: In this study, rTMS was found to be a safe, well-tolerated treatment, and a useful adjunctive treatment to medications in elderly treatment-resistant depressed patients. This study contributes to the existing evidence on the antidepressant effect of rTMS in the treatment of depression in patients over 60 years of age.

Keywords: high-frequency repetitive TMS, rTMS, Hamilton Depression Rating Scale

\section{Introduction}

Among the mental disorders that affect older people, depression is the most frequent cause of reduced quality of life and is recognized as a serious public health problem. ${ }^{1}$ Late-life depression may be due to a variety of factors including longstanding vulnerability, severe stress, and vascular risk. ${ }^{2}$ However, depression in the elderly may be more difficult to treat than in other populations. ${ }^{3-5}$ Antidepressants can be administered as a first-line treatment, but such treatment can be problematic, as older patients often cannot tolerate dosages high enough to produce an antidepressant response. Although electroconvulsive therapy is quite effective and fast acting, it is associated with cognitive and other side effects including nausea, headache, and muscle ache. In addition, due to the risks associated with anesthetic use in the elderly, electroconvulsive therapy is not always a viable option. 
Transcranial magnetic stimulation (TMS) was approved by the US Food and Drug Administration in 2008 as a treatment for pharmacotherapy-resistant depression. ${ }^{6}$ A high-frequency pulse repetitive TMS (rTMS) is applied to the left dorsolateral prefrontal cortex. A 2-week open study examined the antidepressant efficacy of rTMS in vascular depression in an elderly population. ${ }^{7}$ The authors found that $45 \%$ of treatment-resistant patients with lateonset vascular depression were responders. A further two controlled studies carried out in the elderly failed to show a significant effect with active rTMS. ${ }^{8,9}$ Nevertheless, another controlled trial revealed the efficacy of the treatment among geriatric patients with vascular depression. ${ }^{10}$ In that trial, the authors observed response rates of $6.9 \%$ in the sham group and $39.4 \%$ in the active-stimulation group; the remission rates were $3.5 \%$ and $27.3 \%$, respectively. Older age and a smaller volume of frontal gray matter were associated with a poorer response to rTMS. These data suggest that additional studies are required to further assess the efficacy and safety of rTMS as a treatment option for depression in elderly patients.

As such, the aim of the study reported here was to examine the safety and effectiveness of high-frequency rTMS in elderly patients with depression.

\section{Patients and methods}

\section{Study design}

This prospective study was approved by the institutional review board of Uskudar University and the study protocol conformed to the Declaration of Helsinki. Elderly patients (aged over 60 years) who met the Structured Clinical Interview for Diagnostic and Statistical Manual of Mental Disorders, Fourth Edition (DSM-IV) criteria for depression admitted to our clinic between January 2007 and January 2012 were included in the study. "Treatment-resistant depression" was defined as a failure to respond adequately to two successive courses of monotherapy with pharmacologically different antidepressants given in adequate doses for 4-6 weeks.

After obtaining informed consent from subjects, they had a complete neurological and physical examination. Complete blood counts, thyroid function tests, and electrolyte screenings were also performed. Subjects with minor neurological abnormalities (eg, essential tremor, chronic headaches, gait ataxia, prior head injury) underwent neuroimaging, unless they had had prior neuroimaging at the onset of the neurological dysfunction that showed no gross abnormality. Concomitant medication for depression was authorized, provided the dose administered had been stable for at least 1 month before enrollment and remained stable throughout the study.

Exclusion criteria included evidence of dementia on neuropsychological testing, or meeting the Structured Clinical Interview for DSM-IV criteria for organic brain syndrome, organic mood disorder, substance dependence within the last 6 months, a diagnosis of a significant central neurological disorder including brain mass, epileptic seizures, stroke, transient ischemic attack within 2 years, cerebral aneurysm, Parkinson's disease, Huntington's chorea, multiple sclerosis, or any other major central nervous system dysfunction. Additional exclusion criteria included the presence of a cardiac pacemaker, cochlear implant, or other intracranial implant with the exception of dental fillings, and the presence of psychiatric symptoms of significant severity (eg, refusal of food and medication or the presence of psychosis) that would prevent a 3-week trial of rTMS being tolerated. Subjects with acute, unstable medical conditions that required stabilization (eg, uncontrolled hypertension) prior to treatment were also excluded.

\section{Stimulation}

TMS was applied using a Magstim Super Rapid stimulator (Magstim, Whitland, UK) with a figure-of-eight-shaped coil to all patients in an open label manner. The rTMS intensity was set at $100 \%$ of the motor threshold (MT), which was determined by visual inspection. Stimulations were given to the left prefrontal cortex, deemed to be located $5 \mathrm{~cm}$ forward of the cortical motor area of the abductor pollicis brevis from which the MT was determined. The treatment schedule was 6 days per week, from Monday to Saturday for 3 weeks. The $25 \mathrm{~Hz}$ stimulation with duration of 2 seconds was delivered 20 times at 30 -second intervals. A full course comprised 1000 magnetic pulses.

\section{Outcome parameters}

Depressive symptom changes were measured by the validated Hamilton Depression Rating Scale. ${ }^{11}$ The primary outcome parameter, the 17-item Hamilton Depression Rating Scale (HAMD-17) score, constitutes a valid and reliable measure of the severity of depressive symptoms. ${ }^{12}$ HAMD-17 scores were obtained at baseline and 1 week after completing the course of rTMS.

Secondary outcome parameters included response and remission rates. "Response" was defined as a decrease in the HAMD-17 total score of at least 50\%. Patients with HAMD-17 scores of $<8$ were considered to be in remission. 
Efficacy parameters were assessed by an independent rater who was blind to the patient treatment status.

The patients were investigated for side effects such as nausea, headache, muscle ache, cognitive difficulties, seizures, tinnitus, and dizziness.

\section{Statistical analyses}

Data were analyzed using SPSS for Windows (v 16; IBM, Armonk, NY, USA). The results for all items were expressed as mean \pm standard deviation, assessed within $95 \%$ reliance and with values $<0.05$ considered statistically significant.

\section{Results}

A total of 83 elderly patients with depression underwent rTMS treatment from January 2007 to January 2012. Of these 83 patients, 13 were excluded because of associated dementia. Seventy patients signed an informed consent to be enrolled in the study and received the first rTMS session these patients constituted the intention-to-treat population. Five of these 70 patients withdrew from the study. Reasons for withdrawal were unwillingness to come to the hospital repeatedly on a daily basis in four patients and not willing to continue in the study in one patient. None of the patients withdrew due to adverse events.

Prospectively planned data collection of 65 patients who completed the rTMS protocol was retrospectively evaluated. The study population consisted of 42 females and 23 males, with an average of $66.57 \pm 5.77$ (range 60 to 83 ) years. The mean number of rTMS sessions was $18.38 \pm 7.31$ for the study group.

The mean HAMD score for the study group decreased from $21.94 \pm 5.12$ before treatment with rTMS to $11.28 \pm 4.56$ after treatment $(P<0.001)$. After the treatment period, 38 of 65 patients $(58.46 \%)$ demonstrated significant mood improvements, as indexed by a reduction of more than $50 \%$ on the HAMD score. Nineteen of these 38 patients attained remission (HAMD score $<8$ ). Overall, 27 of 65 patients $(41.54 \%)$ achieved a partial response. None of the patients had a worsened HAMD score at the end of treatment.

In our study group, the magnetic stimulation was generally well tolerated. Patients had previously been informed about contractions of facial muscles. Other side effects such as cognitive difficulties, seizures, headache, tinnitus, dizziness, or nausea, which have been observed during rTMS studies in the literature, were not reported in our study group.

\section{Discussion}

This study assessed the effect of rTMS in medicationresistant depressed patients who were 60 years of age or older. After 3 weeks (18 rTMS treatments), 58.46\% (38 out of 65 patients) met response criteria $(>50 \%$ improvement in HAMD score), with 19 of these patients also meeting criteria for remission (HAMD score $<8$ ).

As in previous studies, we used the left prefrontal cortex for stimulation. This localization is based on the historical observation of depression-related hypometabolism of the prefrontal cortex. Herwig et al determined the most hypometabolic cortical areas with positron emission tomography and repetitively localized this area using a stereotaxic coilnavigation system. ${ }^{13}$ We determined stimulation localization with the method initially described by Pascual-Leone et $\mathrm{al}^{14}$ and, to keep variability of stimulation localizations low, most stimulations were applied by the same person.

The results of the present study compare favorably to the studies of Nahas et al and Abraham et al. ${ }^{15,16}$ Both studies used distance-adjusted TMS treatments in patients aged 65-75 years old. Nahas et al administered rTMS for 3 weeks using a stimulation intensity of $114 \%$ of the MT, while Abraham et al used rTMS for 2 weeks at 100\% of MT, which was similar to our study. Mosimann et al treated elderly depressed patients with a shorter treatment period of 10 days with the same $100 \%$ MT and reported a response rate of 26.6\%. ${ }^{9}$ In Nahas et al's study, $28 \%$ met criteria for response; $80 \%$ of these patients had a HAMD score $\leq 8$, and thus met criteria for remission. In Abraham et al's study, the response rate was $30 \%$, with $33 \%$ of these patients also meeting criteria for remission. Our results showed higher response and remission rates when compared with those reported in the literature.

The most common side effects of rTMS treatment are pain or discomfort during stimulation (owing to stimulation of scalp nerves and muscles) and headache, although seizure, facial numbness, and hearing loss have also been rarely reported. ${ }^{9,17}$ Mosimann et al found rTMS to be a safe treatment with none of their patients withdrawing from the study nor reporting serious adverse effects. ${ }^{9}$ In a study by Su et al, three out of 30 patients dropped out of the treatment because of pain or worsening of clinical symptoms, while four patients reported headaches. ${ }^{18}$ In the present study, the magnetic stimulation was generally well tolerated. Our study group did not report the cognitive difficulties, seizures, headache, tinnitus, dizziness, and nausea observed in other rTMS studies in the literature. None of our patients withdrew from the study or reported serious adverse effects. 
Although there are many limitations in the study reported here, such as small sample size and lack of long follow-up, the instrument (Hamilton Depression Rating Scale) used for the outcome measure is specific for depression. Higher responsiveness of specific rather than generic instruments should render the results of this study usable for comparison with studies of other treatment modalities for treatment-resistant depression. The main limitation of this study was the noncontrolled design, which probably restricted the statistical power. Further, since the study lacked a control group, we cannot exclude the possibility of a placebo effect. Further studies are required to define the optimal parameters of stimulation (site, intensity, frequency, etc) so as to clarify which patient and treatment characteristics might lead to a greater antidepressant effect in elderly patients with depression.

\section{Conclusion}

Our study indicates that rTMS is a safe and well-tolerated treatment and may be a useful adjunctive treatment to medications in elderly treatment-resistant depressed patients. We believe our findings add to the growing body of literature that suggests the effectiveness of rTMS. Despite the fact that the generalizability of our study findings is limited, the rTMS treatment of older depressed populations is continuing to show encouraging results and requires further study.

\section{Disclosure}

The authors report no conflicts of interest in this work.

\section{References}

1. Blazer DG. Depression in late life: review and commentary. $J$ Gerontol A Biol Sci Med Sci. 2003;58(3):249-265.

2. O'Connor DW. Do older Australians truly have low rates of anxiety and depression? A critique of the 1997 National Survey of Mental Health and Wellbeing. Aust N Z J Psychiatry. 2006;40(8):623-631.

3. Pallanti S, Cantisani A, Grassi G, et al. rTMS age-dependent response in treatment-resistant depressed subjects: a mini-review. CNS Spectr. 2012;17(1):24-30.

4. Mosimann UP, Marré SC, Werlen S, et al. Antidepressant effects of repetitive transcranial magnetic stimulation in the elderly: correlation between effect size and coil-cortex distance. Arch Gen Psychiatry. 2002;59(6):560-561.
5. Solé-Padullés C, Bartrés-Faz D, Junqué C, et al. Repetitive transcranial magnetic stimulation effects on brain function and cognition among elders with memory dysfunction. A randomized sham-controlled study. Cereb Cortex. 2006;16(10):1487-1493.

6. Andreescu C, Reynolds CF 3rd. Late-life depression: evidence-based treatment and promising new directions for research and clinical practice. Psychiatr Clin North Am. 2011;34(2):335-355, vii-iii.

7. Fabre I, Galinowski A, Oppenheim C, et al. Antidepressant efficacy and cognitive effects of repetitive transcranial magnetic stimulation in vascular depression: an open trial. Int J Geriatr Psychiatry. 2004;19(9): 833-842.

8. Manes F, Jorge R, Morcuende M, et al. A controlled study of repetitive transcranial magnetic stimulation as a treatment of depression in the elderly. Int Psychogeriatr. 2001;13(2):225-231.

9. Mosimann UP, Schmitt W, Greenberg BD, et al. Repetitive transcranial magnetic stimulation: a putative add-on treatment for major depression in elderly patients. Psychiatry Res. 2004;126(2):123-133.

10. Jorge RE, Moser DJ, Acion L, Robinson RG. Treatment of vascular depression using repetitive transcranial magnetic stimulation. Arch Gen Psychiatry. 2008;65(3):268-276.

11. Hamilton M. A rating scale for depression. J Neurol Neurosurg Psychiatry. 1960;23:56-62.

12. Akdemir A, Örsel S, Dağ İ, Türkçapar H, İşcan N, Özbay H. Hamilton depresyon derecelendirme ölçeği (HDDÖ) 'nin geçerliliği, güvenilirliği ve klinikte kullanımı [Hamilton Depression Rating Scale (HDRS) and its validity, reliability and clinical use]. Psikiyatri Psikoloji Psikofarmakoloji Dergisi. 1996;4(4):251-259. Turkish.

13. Herwig U, Lampe Y, Juengling FD, et al. Add-on rTMS for treatment of depression: a pilot study using stereotaxic coil-navigation according to PET data. J Psychiatr Res. 2003;37(4):267-275.

14. Pascual-Leone A, Rubio B, Pallardó F, Catalá MD. Rapid-rate transcranial magnetic stimulation of left dorsolateral prefrontal cortex in drug-resistant depression. Lancet. 1996;348(9022):233-237.

15. Nahas Z, Li X, Kozel FA, et al. Safety and benefits of distance-adjusted prefrontal transcranial magnetic stimulation in depressed patients 55-75 years of age: a pilot study. Depress Anxiety. 2004;19(4): 249-256.

16. Abraham G, Milev R, Lazowski L, Jokic R, du Toit R, Lowe A. Repetitive transcranial magnetic stimulation for treatment of elderly patients with depression - an open label trial. Neuropsychiatr Dis Treat. 2007;3(6):919-924.

17. Janicak PG, O'Reardon JP, Sampson SM, et al. Transcranial magnetic stimulation in the treatment of major depressive disorder: a comprehensive summary of safety experience from acute exposure, extended exposure, and during reintroduction treatment. J Clin Psychiatry. 2008;69(2):222-232.

18. Su TP, Huang CC, Wei IH. Add-on rTMS for medication-resistant depression: a randomized, double-blind, sham-controlled trial in Chinese patients. J Clin Psychiatry. 2005;66(7):930-937.
Neuropsychiatric Disease and Treatment

\section{Publish your work in this journal}

Neuropsychiatric Disease and Treatment is an international, peerreviewed journal of clinical therapeutics and pharmacology focusing on concise rapid reporting of clinical or pre-clinical studies on a range of neuropsychiatric and neurological disorders. This journal is indexed on PubMed Central, the 'PsycINFO' database and CAS.

\section{Dovepress}

The manuscript management system is completely online and includes a very quick and fair peer-review system, which is all easy to use. Visit http://www.dovepress.com/testimonials.php to read real quotes from published authors. 